\title{
Meditación sobre la compleja relación entre medicina y animales: un responso para un gato fiel
}

\author{
FELIPE CABELLO C.
}

\section{Thoughts on the complex relationship between medicine and animals: a death prayer for a loyal cat}

From its basis in the writings of the philosopher Peter Singer and the bioethical shortcomings of animal experimentation and animal husbandry, the animal rights movement has evolved into an important societal movement critical of animal experimentation in biomedical research. A lack of dialogue and transparency, an absence of understanding and an unreasonable radicalization of different positions regarding animal experimentation has frequently resulted in an adversarial relationship between some members of the scientific community and societal groups aggressively protecting animal rights. In response to this problem, both the bioethical regulations pertaining to biomedical experimentation with animals and the powers of animal care committees (IACUCS) have been strengthened. Careful analysis of the relevance of animal models to human conditions, replacement of these models with non-animal models when possible, adequate re-examination of existing knowledge before undertaking new experimental projects involving animals, and the improvement of methods to avoid animal stress and pain have further strengthened the bioethical basis of animal experimentation. To improve the ethical integrity of research conducted with animals, it is also necessary to increase the editorial scrutiny of the bioethical standards of potentially publishable research utilizing animals. Of note is also the recent use of animals in alternative animal associated therapies (AAT) to ameliorate several medical conditions. Education of the biomedical community, including students and professionals, and of societal groups concerned about this issue as well as directness and continuous dialogue among all the stakeholders are essential to insure the wellbeing of animals and the ethical integrity of biomedical research.

(Rev Med Chile 2013; 141: 1449-1455)

Key words: Animal experimentation; Animal welfare; Bioethics; Models, animal.

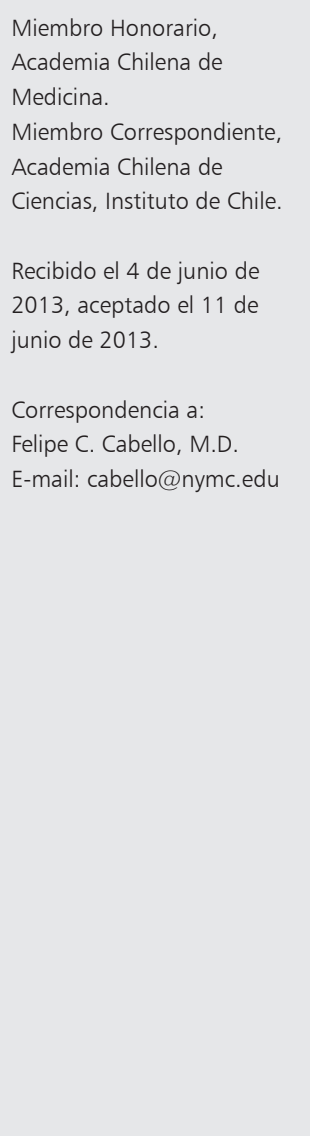

"Si tenemos compasión por el sufrimiento de los animales, tendremos, una similar o aun mayor compasión y simpatía por el sufrimiento de nuestros semejantes".

Fritz Jahr, 1928.

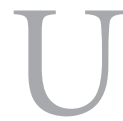
na tarde de verano del año 1995 llegaban a mis oídos a través de la ventana abierta, interrumpiendo la tranquilidad de la tarde, los maullidos quejumbrosos y lejanos de un gato. Por ello, cuando más tarde escuché a mi esposa abriendo la puerta de la casa y diciéndome en tono festivo, “¿adivina qué traigo?”, le contesté de manera inmediata e intuitiva, “ $¡ u n$ gato!” De esta manera espontánea se incorporaron a nuestra rutina diaria las interacciones con un juvenil felino, compartidas más de diecisiete años (Figura 1). Esta relación, que dio origen a múltiples y recíprocas satisfacciones y que también en algunas ocasiones se acompañó de angustias, se convirtió 
además en una fuente permanente de estímulo para reflexionar acerca de las relaciones entre humanos y animales.

Durante mis peregrinaciones laborales por los Estados Unidos de Norteamérica, Canadá y Alemania siempre me he sentido extraordinariamente orgulloso de la educación y de la formación profesional que recibí en la Escuela de Medicina de la Universidad de Chile. Porque ella, a mi juicio, fue comparable en calidad y en muchos aspectos superior, a la de las mejores Universidades del mundo de esa época, incluyendo por ejemplo Harvard, Stanford y Oxford. Este gran orgullo se vio parcialmente opacado con la entrada a mi vida de nuestro compañero gato, "Kitty" en su juventud y "Mr. Cat" durante su adultez. Esto porque en mis tratos con él, desde que me despertaba de madrugada con sus maullidos plañideros o saltaba a mi cama para encontrar caricias y un lugar tibio donde refugiarse, hasta que yo le daba sus últimas golosinas nocturnas, me hacía recordar de una manera culpable, los ingratos momentos que compartí con otros infortunados felinos en los primeros años de mi educación médica.

El uso de animales de experimentación era una práctica común y difundida en los años 1960 en las escuelas de medicina del mundo y era también una práctica habitual en Chile. De esta manera como alumnos experimentábamos y se nos hacían demostraciones pedagógicas con ratas, gatos, perros, cobayos y conejos durante los tres primeros años de la educación médica. Los crueles

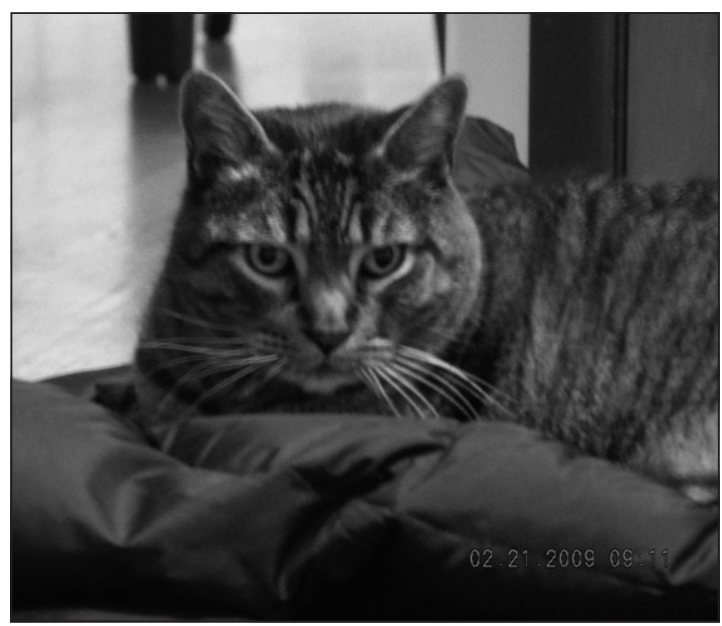

Figura 1. Mr. Cat. e innecesarios experimentos que mi gato me hacía recordar, eran aquellos destinados a ver la función de mediadores del sistema nervioso sobre la contracción de la membrana nictitante del ojo de los felinos. Estos experimentos requerían de anestesia general del animal seguida de su inmovilización, de la denudación quirúrgica de una de las carótidas y de la perforación de la nictitante ipsilateral con un garfio para registrar la contracción de ella en un quimógrafo; continuado esto con la inyección intracarotídea de acetil colina, muscarina y pilocarpina. Estos experimentos eran llevados a cabo sin la suficiente instrucción, con anestesias a menudo superficiales, en inadecuadas condiciones de asepsia y además la impericia quirúrgica de los estudiantes obligaba innecesariamente a sacrificar a dos o tres felinos, antes de llegar a tener una preparación adecuada.

Los procedimientos de eutanasia de estos animales tampoco eran apropiados, ya que no aseguraban su total inconciencia y la ausencia de dolor, generándose en mi opinión retrospectiva una práctica cruel y carente de ética para con los animales de experimentación. Tal vez lo más perjudicial de estos experimentos era el uso dispendioso de un número excesivo de animales, lo cual podría haber sido evitado con una apropiada instrucción y prolijidad docente. En el transcurso de mi vida profesional he realizado experimentación con varios animales, con diversas bacterias y en diferentes modelos de infección. Como experimentador y miembro de comités institucionales destinados a regular el uso y a vigilar el bienestar de los animales de experimentación, aprendí a apreciar las limitaciones y las regulaciones que impulsan ellos y las instituciones que patrocinan investigación en ciencias biomédicas, con el propósito de salvaguardar el uso éticamente correcto de estos animales ${ }^{1}$. Sin lugar a dudas esta manera de ver el problema se vio importantemente reforzada por la convivencia diaria con nuestro gato durante los últimos diecisiete años y por las deliberaciones, interrogantes y satisfacciones que esta cotidiana y beneficiosa convivencia impulsó.

Las primeras críticas en contra de los fundamentos éticos que sustentarían la experimentación biomédica en animales surgieron en Inglaterra durante el Siglo XIX, destacando, entre otros, las del filósofo y economista Jeremy Bentham y las del autor de "Alicia en el país de las maravillas" Charles L. Dogson (Lewis Carrol) ${ }^{1,2,3}$. Sin embargo, 
éstas comenzaron a globalizarse y crecer en los años 1960, paralelamente y tal vez influenciadas por el escrutinio crítico que durante los juicios de Nuremberg se hiciera de la experimentación biomédica en humanos, realizada por el régimen nazi en Alemania ${ }^{4}$. Las debacles éticas que significaron también los famosos experimentos con pacientes sifilíticos en Tuskegee y con pacientes pediátricos limitados psicológicamente en Willowbrook, en los EE. UU., y el debate público que estimularon, ayudaron a generar una mirada con una nueva sensibilidad más indagadora y sofisticada de la experimentación con animales ${ }^{5-7}$. De esta manera, en el año 1975 el ahora profesor de bioética de la Universidad de Princeton, Peter Singer, publicó la clásica obra titulada "Liberación animal"2 En ella hizo un análisis histórico detallado, que abarca desde la Antigüedad hasta el presente, de los fundamentos éticos, filosóficos, religiosos y científicos que guiaron las interacciones humanas con los animales, de cómo han variado a través de la historia y de cómo habían sido en general guiadas por el prejuicio y la ignorancia, representándose habitualmente a los animales como seres inferiores y carentes de sensibilidad ${ }^{2}$. El profesor Singer concluyó en su obra que los animales tendrían derechos inherentes que deberían ser respetados de la misma forma en que se respetan los derechos humanos y que estos derechos habrían sido prejuiciada e irracionalmente ignorados ${ }^{2}$. En la obra el autor hizo suyo el planteamiento de Jeremy Bentham acerca de que en las relaciones humanas con los animales "...la pregunta adecuada no es si los animales razonan o pueden hablar, sino es si ellos sufren...", respondiendo afirmativamente y sin vacilaciones a esta pregunta, al igual que previamente lo había hecho J. Bentham ${ }^{2,8}$.

Coincidiendo con la publicación de esta seminal y debatida obra, aumentaron también las revelaciones y las críticas de una gran variedad de experimentos con animales que se demostraban como de escasa utilidad para el desarrollo de las ciencias biomédicas, además de estar mal planificados y de llevarse a cabo sin las mínimas precauciones para evitar el sufrimiento animal ${ }^{9}$. Estas transformaciones coincidieron también con la diseminación de información respecto de las terribles condiciones ambientales y sanitarias en las que se desarrollaba la industria productora de animales como alimento para consumo humano: aves, cerdos y vacunos ${ }^{10}$. Además de la publicidad negativa respecto de la utilización de animales para ensayar la inocuidad de cosméticos en esa industria, de manera mecánica y desregulada ${ }^{11}$. Como resultado de la difusión de esta negativa información, innumerables organizaciones ciudadanas y de profesionales, incluyendo los de la salud, comenzaron a ejercer presión política a nivel de parlamentos y gobiernos y de las instituciones patrocinadoras de investigaciones biomédicas, para definir y garantizar las bases bioéticas y la ausencia de sufrimiento en los experimentos llevados a cabo con animales ${ }^{12}$.

Desgraciadamente, algunos investigadores interpretaron estos esfuerzos como una limitación a la libertad de investigación y una intromisión injustificada de legos en su trabajo y reaccionaron de manera antagónica a ellos ${ }^{13}$. Además, grupos ciudadanos y de profesionales sorprendidos por la crueldad y la demostrada inutilidad de algunos de estos experimentos y la negativa de un importante número de investigadores a reconocerlo, radicalizaron sus demandas para limitar drásticamente la experimentación biomédica con animales ${ }^{14-17}$. Estos perjudiciales procesos afortunadamente han evolucionado en los últimos años de una manera positiva y ha habido un reconocimiento por parte de la comunidad científica que deben existir limitaciones bioéticas a la experimentación con animales y los grupos ciudadanos han comenzado a reconocer que en algunas circunstancias la experimentación con animales pareciera ser una necesidad para el progreso de la medicina ${ }^{12,18}$. El reconocimiento del concepto desarrollado por el profesor Singer y otros, de que existen animales que tienen conciencia (seres sintientes) similar a la de los humanos ha sido importante para construir una bioética que fundamente y regularice la

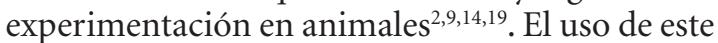
concepto, por ejemplo, ha sido fundamental para que los Institutos Nacionales de la Salud (NIH), Estados Unidos de Norteamérica, asesorados por el Instituto de Medicina (IOM), recientemente eliminaran prácticamente la investigación con chimpancés, cuyo grado de conciencia y de elaboración mental al parecer nadie discute ${ }^{20,21}$.

La habilidad de un animal de interpretar los estímulos de su ambiente interno y externo y de tener memoria, manifestar emociones y tener la capacidad de elegir entre diversas posibilidades es lo que el profesor Singer definió como "conciencia" y es lo que le hizo concluir que los animales tienen 
derechos que deberían ser respetados ${ }^{1,2,9,19}$. Esto sin lugar a dudas es confirmado por las observaciones de Darwin, quien indicó que los animales tienen cierta capacidad de razonar ${ }^{22}$. Como él ya lo observara, y yo lo hiciera diariamente con "Mr. Cat", los animales en el proceso de efectuar una acción son capaces de detenerse, esperar y tal vez pensar y resolver en seguida continuar con ella o abandonarla ${ }^{22}$. Sin lugar a dudas el origen común de los seres humanos y de los animales, de acuerdo a la teoría de la evolución, explicaría estas similitudes en nuestro comportamiento y este origen común es también una razón más aducida para respetar los derechos de los animales, en general, y por supuesto también los derechos de los animales sujetos de experimentación ${ }^{2,23}$. A pesar de este origen común, las importantes diferencias entre la biología de los animales usados como modelos de diversas enfermedades humanas y la biología humana es otra razón más para morigerar el uso de ellos en estas actividades, ya que según los postuladores de esta hipótesis estos experimentos que sacrifican animales tendrían escasa aplicabilidad y utilidad para la especie humana ${ }^{24,25}$. Similarmente, algunos autores postulan que la experimentación en animales estaría, además, limitada por el imperativo bioético, que sería una continuación del imperativo moral de la filosofía de Kant, de no producir daño a seres vivientes y que fuera inicialmente postulado por el teólogo alemán Fritz Jahr a comienzos del Siglo XX ${ }^{26,27}$.

Está claro que los crueles experimentos de François Magendie durante el Siglo XIX fueron fundamentales para identificar la ubicación en la médula espinal de las raíces ventrales motoras y de las dorsales sensoriales, pero al mismo tiempo horrorizaron a sus contemporáneos por su brutalidad y fueron una de las razones que estimularon las primeras campañas en contra de la experimentación con animales ${ }^{13,28}$. Similarmente, los experimentos en ratones con Streptococcus pneumoniae de F. Griffith, abrieron la puerta a los experimentos de O. T. Avery y cols demostrando la naturaleza química (ADN) del material genético ${ }^{29,30}$. Sin embargo, esto no significa que todos los experimentos con animales avancen de alguna forma el progreso de las ciencias biomédicas ${ }^{25,31}$. Lo relevante de esta consideración en la planificación de experimentos con animales ha sido recientemente demostrada al parecer por la total diferencia con que reaccionan humanos y ratones a los estímulos nocivos que producen shock séptico ${ }^{32-34}$. Esto ha hecho concluir a científicos y legos que durante los últimos 50 años, como lo decía un artículo reciente del New York Times, se han sacrificado inútilmente a millones de ratones y se han gastado billones de dólares en experimentos improductivos ${ }^{33}$. Esto porque la manera en que humanos y ratones reaccionan frente a las noxas que desencadenan el shock séptico son totalmente diferentes, generando el modelo animal con ratones, información que es irrelevante al problema humano ${ }^{32-34}$. Resultados como éstos y la escasa aplicabilidad reciente de la investigación básica con animales a la solución directa de problemas clínicos, ha hecho que se levanten voces en la comunidad científica diciendo que los experimentos con animales debieran ser drásticamente limitados ${ }^{15-18,35,36}$.

Por todas estas facetas y los problemáticos aspectos de la experimentación con animales, es que además de la instauración de comités y de regulaciones para vigilar la integridad ética de estos procesos, se ha introducido también el concepto ético de "las 3R", abreviatura que corresponde a "reemplazo, reducción y refinamiento"1,37. En cada experimento que usa animales se debería tratar de optimizar el remplazo de experimentos con animales por otros que no los utilicen, se debiera tratar de reducir el número de animales utilizados para obtener idénticas conclusiones y debiera haber un refinamiento de las técnicas usadas para evitarles un sufrimiento innecesario ${ }^{1,37}$. Está claro que en el proceso de elevar los niveles bioéticos de la experimentación en animales tienen un rol diversos estamentos, además de los científicos, y últimamente se ha señalado que un número importante de investigaciones con animales son publicadas sin que durante su proceso editorial los editores se hayan asegurado de la integridad bioética de los experimentos descritos en ellas ${ }^{38,39}$. Indudablemente que este tipo de limitaciones debiera ser corregido, ya que todo resultado científico publicado debiera ejecutarse con integridad bioética y, además, porque estos lapsos son los que generan desconfianza en los estamentos legos de la sociedad acerca de la habilidad de la comunidad científica para autorregularse respecto de estos problemas $^{38,39}$. Las revistas científicas tendrían que asegurarse de que, antes de su publicación, los artículos remitidos a ellas y que incluyan experimentos con animales, cumplan los alineamientos sugeridos por las pautas reguladoras de ARRIVE 
(Animal Research Reporting in vivo Experiments) ${ }^{39}$.

Estas inquietudes y reflexiones que, en mi caso, han sido motivadas por experiencia propia, al comienzo desafortunada con la experimentación con animales, y por las fructíferas relaciones con un felino regalón, deberían ser también estimuladas durante la educación médica de pre y post grado, ya que todo relativismo ético y la crueldad con los animales tienen indirectamente el potencial de afectar negativamente nuestra virtud, como lo dijera F. Jahn en la cita que inicia este artículo $^{26,27}$. Teniendo la posibilidad de afectar nocivamente la integridad ética de la relación de los médicos con sus pacientes, como lo sugiriera premonitoriamente Lewis Carrol hace ya más de un siglo ${ }^{3,26,27}$. Como partes importantes de la sociedad tienen, además, una visión negativa acerca del uso de animales en experimentación biomédica, especialmente cuando la relación entre este uso y el progreso de la medicina es tenue o aun inexistente, en la educación médica a todos los niveles debería hacerse un esfuerzo constante para impartir a los estudiantes y a los profesionales los conceptos bioéticos fundamentales de esta práctica, como una manera de prevenir conflictos sociales futuros ${ }^{1,14,18}$. Pareciera ser de sentido común que el uso de animales primariamente como objetos es inadecuado y que, por ejemplo, en nuestras relaciones con ellos debiéramos aplicar similares juicios de valor a un gato por ser un gato y no porque sea en algunas circunstancias un animal regalón, un animal de experimentación, una fuente de piel para ropa o el origen de una zoonosis ${ }^{14}$.

Es importante destacar también que, a medida que ha habido un cambio en las sensibilidades éticas respecto del uso de los animales de experimentación en medicina y de la crianza industrial de animales para alimento humano ${ }^{2,10}$, se ha producido en medicina un cambio positivo en estas relaciones con el uso de animales como adyuvantes teraupeuticos ${ }^{40,41}$. De esta forma, en la Norte América anglosajona y en Europa ha aparecido una nueva forma terapéutica alternativa llamada terapia asistida con mascotas que consiste en la exposición de pacientes a la interacción con ellas, con el objeto de mejorar sus estados de ánimo y acelerar la recuperación de diversas patologías $^{40,41}$. En la literatura científica se encuentra un número creciente de publicaciones respecto al uso de animales como coadyuvantes en técnicas para combatir el estrés, el dolor y la depresión ${ }^{40,41}$. A cualquier amo de mascotas regalonas que ha experimentado, como yo experimenté diariamente con nuestro gato, el efecto tangible de aliviar tensiones, de distracción, de empatía recíproca y de estimular la imaginación que el contacto psicológico y físico con un animal consentido provoca, puede entender el efecto benéfico de esta terapia ${ }^{40,41}$. El origen biológico común que tenemos con los animales y el hecho de que ellos al parecer tengan conciencia, son factores que agregan más firmeza aún a las bases biológicas de ella ${ }^{19,22,23}$. Optimista y generosamente se pudiera esperar que tal vez en el futuro los animales puedan contribuir más a la medicina como agentes terapéuticos que como sujetos involuntarios de experimentación. O tal vez como partes del equipo de salud, como el gato Oscar, cuyas hazañas y fotos aparecieran en el New England Journal of Medicine y en muchos medios de difusión estadounidenses, gracias a su habilidad en predecir la muerte de los pacientes en un hogar de ancianos y, además, ayudar a los familiares en este trance ${ }^{42}$.

Diálogos, transparencia y educación acerca de este problema parecieran ser muy necesarios en países como Chile y en otros países Latino Americanos, donde un catastro reciente ha encontrado importantes falencias en los procesos que aseguren la integridad ética de la experimentación con animales ${ }^{1}$. Para finalizar esta breve meditación me gustaría citar al médico alemán Albert Schweitzer (Premio Nobel de la Paz, 1952), quien dijo que los individuos que experimentan con animales “jamás debieran estar totalmente satisfechos con la generalizada idea de que este tipo de actividades son siempre benignas y que favorecen el bien común..."43, y es este justamente un concepto que debiera ser infundido en el acervo de la educación médica. Otro planteamiento que también estimula a la reflexión en este campo es el del naturalista estadounidense Henry Beston, quien dice "...Los animales no deben ser comparados con los humanos... Los animales no son nuestros hermanos ni tampoco nuestros inferiores, ellos son otras naciones, apresadas junto con nosotros en la redes de la existencia y del tiempo, frutos del esplendor y de las labores de la vida en la tierra..."

Agradecimientos: Agradezco a mi esposa, Lilo Aron, la adopción de nuestro gato y el esfuerzo y el cuidado generoso para mantenerlo saludable y contento a través de su larga vida. Agradezco 
también la compañía de nuestro gato, "Kitty" o "Mr. Cat", ya que él fue un inseparable amigo y un diario incentivo intelectual y físico para ponderar sobre las relaciones entre humanos, animales y la naturaleza, además de ser un bálsamo en época de vicisitudes y tiempos difíciles. Agradezco a mi institución empleadora, New York Medical College, por confiarme con la oportunidad de ser miembro de diversos comités que vigilan el bienestar de los animales de experimentación. La estimulante interacción con los miembros del Literature Selection Technical Review Committee (LSTRC), National Library of Medicine, National Institutes of Health, U. S. A., ha contribuido a aguzar mi sensibilidad y discernimiento frente a los problemas éticos generados en la práctica de la medicina, por los conflictos de intereses y la experimentación en humanos y en animales. La Sra. H. Harrison contribuyó eficientemente a la preparación del manuscrito.

\section{Referencias}

1. Cardozo de Martínez A, Mrad de Osorio A, Martínez C. Rodríguez-Yunta E, Lolas-Stepke F. El animal como sujeto experimental. Aspectos técnicos y éticos. 2007. Centro Interdisciplinario de Estudios en Bioética (CIEB). Vicerrectoría de Investigación y Desarrollo. Universidad de Chile. Santiago, Chile.

2. Singer P. Animal Liberation. A New Ethics for Our Treatment of Animals. New York: Avon Books. The Hearst Corporation, 1975.

3. Lewis Carrol. Some popular fallacies about vivisection. Fornightly Review. 1875. Jun. 847-854. http://www. animalrightshistory.org/animal-rights-c1837-1901/ victorian-c/car-lewis-carroll/1875-06-vivisection.htm

4. Shuster E. The Nuremberg Code: Hippocratic ethics and human rights. Lancet 1998; 351: 974-77.

5. Becker GJ. Human subjects investigation: timeless lessons of Nuremberg and Tuskegee. J Am Coll Radiol 2005; 2: 215-7.

6. Rothman DJ. Were Tuskegee \& Willowbrook 'studies in nature'? Hastings Cent Rep 1982; 12: 5-7.

7. Rothman DJ. Research ethics at Tuskegee and Willobrook. Am J Med 1984; 77: A49.

8. Editorial. Animals in research: “can they suffer?" Lancet 2011; 378: 289.

9. Singer P. All animals are equal. In: Regan T, Singer P, eds. Animal Rights and Human Obligations, New Jersey: Prentice-Hall 1989; pp. 73-86.

10. Singer P. Down on the factory farm. In: Singer P, ed.,
Animal Liberation. A New Ethics for Our Treatment of Animals. New York: Avon Books. The Hearst Corporation, 1975. pp. 159-68.

11. Abbott A. More than a cosmetic change. Nature 2005; 438: 144-6.

12. Bayvel ACD, Cross N. Animal welfare: A complex domestic and international public-policy issue-Who are the key players? J Vet Med Ed 2010; 37: 3-12.

13. Gallistel CL. Bell, Magendie, and the proposal to restrict the use of animals in neurobehavioral research. Amer Psychol 1981; 36: 357-60.

14. Broom DM. A history of animal welfare science. Acta Biotheor 2011; 59: 121-37.

15. Greek R, Greek J. Is the use of sentient animals in basic research justifiable? Philos Ethics Humanit Med 2010; 5 : 14. doi: 10.1186/1747-5341-5-14.

16. Rossi J. Toward a zoocentric animal ethics. Am J Bioethics 2008; 8: 50-2.

17. Nobis N. The harmful, nontherapeutic use of animals in research is morally wrong. Am J Med Sci 2011; 342: 297-304.

18. Festing S. Animal experimentation: The need for deliberation and challenge. Altern Lab Anim 2008; 36: 1-4.

19. Silverman J. Sentience and sensation. Lab Animal 2008; 37: 465-7.

20. Gorman J. U.S. will not finance new research on chimps. NY Times 2011; http://www.nytimes.com/2011/12/16/ science/chimps-in-medical-research.html

21. Wadman M. US chimpanzee research to be curtailed. Most biomedical research on chimpanzees deemed 'unnecessary'. Nature 2011; http://www.nature.com/news/ us-chimpanzee-research-to-be-curtailed-1.9.

22. Darwin C. Comparison of the mental powers of man and the lower animals. In: Regan T, Singer P, eds. Animal Rights and Human Obligations. Second edition. New Jersey: Prentice-Hall 1989; pp. 27-31.

23. Shanks N, Pyles RA. Evolution and medicine: the long reach of "Dr. Darwin". Philos Ethics Humanit Med 2007; 2: 4. (http://www.peh-med.com/content/2/1/4).

24. Greek R. Patients are not rodents writ large. Am J Med Sci 2011; 342: 345.

25. Knight A. Systematic reviews of animal experiments demonstrate poor contributions toward human healthcare. Rev Recent Clin Trials 2008; 3: 89-96.

26. Sass H. Fritz Jahr's 1927 concept of bioethics. Kennedy Institute of Ethics Journal 2008; 17: 279-95

27. Lolas F. Bioethics and animal research. A personal perspective and a note on the contribution of Fritz Jahr. Biol Res 2008; 41: 119-23.

28. Tubbs RS, Loukas M, Shoja MM, Shokouhi G, Oakes WJ. François Magendie (1783-1855) and his contributions 
Animales, medicina y bioética - F. Cabello

to the foundations of neuroscience and neurosurgery. J Neurosurg 2008; 108: 1038-42.

29. Griffith F. The significance of pneumococcal types. J Hyg 1928; XXVII: 113-59.

30. Lederberg J. The transformation of genetics by DNA: An anniversary celebration of Avery, MacLeod and McCarty (1944). Genetics 1994; 136: 423-6.

31. Hackam DG, Redelmeier DA. Translation of research evidence from animals to humans. JAMA 2011; 296: 1731-2.

32. Editorial. Of men, not mice. Nature Med 2013; 19: 379. http://www.nature.com/nm/journal/v19/n4/full/ nm.3163.html?WT.ec_id=NM-201304

33. Kolata G. Mice fall short as test subjects for some of humans' deadly ills. http://www.nytimes.com/2013/02/12/ science/testing-of-some-deadly-diseases-on-mice-mislead-report-says.html?pagewanted $=$ all\&_r $=0$. NYTimes February 11, 2013.

34. Seok J, Warren HS, Cuenca AG, Mindrinos MN, Baker $\mathrm{HV}, \mathrm{Xu} \mathrm{W}$, et al. Genomic responses in mouse models poorly mimic human inflammatory diseases. Proc Natl Acad Sci U S A 2013: 110: 3507-12.

35. Knight A. Reviewing existing knowledge prior to conducting animal studies. Alter Lab Anim 2008; 36: 709-12.

36. Stephens M. Animal research: replacing the lab rat. Nature 2011; 471: 449.
37. Blakemore C, MacArthur Clark J, Nevalainen T, Oberdorfer M, Sussman A. Implementing the 3Rs in neuroscience research: A reasoned approach. Neuron 2012; 75 : 948-50.

38. Kilkenny C, Parsons N, Kadyszewski E, Festing MFW, Cuthill IC, Fry D, et al. Survey of the quality of experimental design, statistical analysis and reporting of research using animals. PLoS One 2009; 4: e7824.

39. Kilkenny C, Brown WJ, Cuthill IC, Emerson M, Altman DG. Improving bioscience research reporting: The ARRIVE guidelines for reporting animal research. PLoS Biol 2010; 8: e1000472.

40. Abate SV, Zucconi M, Boxer BA. Impact of canineassisted ambulation on hospitalized chronic heart failure patients' ambulation outcomes and satisfaction. A pilot study. J Cardiovasc Nurs 2011; 26: 224-30.

41. Marcus DA. The science behind animal-assisted therapy. Curr Pain Headache Rep 2013; 17: 322.

42. Dosa DM. A day in the life of Oscar the cat. N Engl J Med 2007; 357: 328-9.

43. Schweitzer A. The ethic of reverence for life. In: Regan T, Singer P, eds. Animal Rights and Human Obligations. New Jersey: Prentice-Hall 1989; pp. 32-37.

44. Beston H. The Outermost House: A Year of Life On The Great Beach of Cape Cod. 1928. Reprint 2003. Henry Holt and Company. New York. 